\title{
Erratum to: Appendectomy in Germany-an analysis of a nationwide survey 2011/2012
}

\author{
Dirk Rolf Bulian • Jürgen Knuth • Axel Sauerwald • \\ Michael Alfred Ströhlein • Rolf Lefering • Jörg Ansorg • \\ Markus Maria Heiss
}

Published online: 22 September 2013

(C) Springer-Verlag Berlin Heidelberg 2013

\section{Erratum to: Int J Colorectal Dis (2013) 28: 127-138 DOI 10.1007/s00384-012-1573-9}

The original version of this article was unfortunately published with errors in Table 3. There were six lines missing in it, which reflects a completely different result. We apologize to our readers for leading to confusion on the interpretation of the results. The correct version of Table 3 is presented below:

Table 3 (Peri)operative details for open (OA) and laparoscopic (LA) appendectomy

\begin{tabular}{lcc}
\hline Variable & OA (\%) & LA (\%) \\
\hline Antibiotics & & \\
$\quad$ General therapy & 1.4 & 0.9 \\
$\quad$ Antibiotic prophylaxis & 55.1 & 56.2 \\
$\quad$ Depending on intraoperative finding & 43.4 & 42.6 \\
$\quad$ Never & 0.2 & 0.3 \\
Appendix & & \\
$\quad$ Ligation & 93.8 & n.a. \\
& &
\end{tabular}

The online version of the original article can be found at http://dx.doi.org/ 10.1007/s00384-012-1573-9.

D. R. Bulian $(\bowtie) \cdot J$. Knuth $\cdot$ M. A. Ströhlein $\cdot$ M. M. Heiss Department of Abdominal, Vascular and Transplant Surgery, Cologne-Merheim Medical Center, Witten/Herdecke University, Ostmerheimer Strasse 200, 51109 Cologne, Germany

e-mail: buliand@kliniken-koeln.de

\section{A. Sauerwald}

Department for Obstetrics and Gynaecology Holweide,

Neufelder Str 32, 51067 Cologne, Germany

\section{R. Lefering}

Institute for Research in Operative Medicine, Witten/Herdecke University, Ostmerheimer Strasse 200, 51109 Cologne, Germany

\section{J. Ansorg}

Professional Association of German Surgeons (BDC),

Berlin, Germany
Table 3 (continued)

\begin{tabular}{lcc}
\hline Variable & OA (\%) & LA $(\%)$ \\
\hline Stapling device/endo-GIA & 3.6 & 66.6 \\
Endoloop only & n.a. & 24.2 \\
Endoloop with purse string/Z-suture & n.a. & 2.6 \\
Resorbable clips & 2.2 & 3.8 \\
Nonresorbable clips & 0.3 & 1.2 \\
Mesoappendix & & \\
Ligation & 91.5 & n.a. \\
Bipolar coagulation & 6.1 & 45.5 \\
Stapling device & 1.2 & 12.5 \\
Resorbable clips & 0.9 & 15.1 \\
Nonresorbable clips & 0.3 & 9.2 \\
Monopolar coagulation & n.a. & 6.4 \\
Lavage routinely & & \\
Yes & 48.1 & 49.9 \\
No & 51.9 & 50.1 \\
Abdominal drain & & n.a. \\
Always/mostly & 9.5 & n.a. \\
Rarely/never & 21.4 & 3.3 \\
Depending on intraoperative finding & 69.1 & 57.5 \\
Subcutaneous Redon drain & & \\
Always/mostly & 3.5 .3 \\
Rarely/never & 75.5 \\
Depending on intraoperative finding & 19.9 & \\
Subcutaneous suture & & \\
Always/mostly & 42.6 & \\
Rarely/never & 42.4 & \\
Depending on intraoperative finding & 15 & \\
Skin closing & & \\
Intracutaneous and resorbable & & \\
Interrupted sutures & & \\
Staples & & \\
Intracutaneous and non resorbable & & \\
\hline
\end{tabular}

n.a. not applicable 